\title{
The Prediction Analysis of Autistic and Schizotypal Traits in Attentional Networks
}

\author{
Wanling Huang ${ }^{1 *}$, Long Zhang ${ }^{2,3,4,5 *}$, Yaoting Sun ${ }^{6 *}$, Fangfang Chen ${ }^{7}$, and Kai Wang ${ }^{2,3,4,5} \bowtie$ \\ ${ }^{1}$ Department of Neurology, Yangpu Hospital, Tongji University School of Medicine, Shanghai, China \\ ${ }^{2}$ Department of Neurology, The First Affiliated Hospital, Anhui Medical University, Hefei, China \\ ${ }^{3}$ Anhui Province Key Laboratory of Cognition and Neuropsychiatric Disorders, Hefei, China \\ ${ }^{4}$ Collaborative Innovation Center of Neuropsychiatric Disorders and Mental Health, Hefei, China \\ ${ }^{5}$ Department of Medical Psychology, The First Affiliated Hospital, Anhui Medical University, Hefei, China \\ ${ }^{6}$ Key Laboratory of Xin'an Medicine, Ministry of Education, Anhui University of Chinese Medicine, Hefei, China \\ ${ }^{7}$ The Fourth People's Hospital of Wuhu, Wuhu, China
}

\begin{abstract}
Objective Empirical findings confirmed that autistic and schizotypal traits are associated with attentional function as well as include various dimensions. So far, no study has reported which dimension of these traits relates to attentional networks. This study aimed to find out whether there are associations between attentional networks and autistic traits; and between attentional networks and schizotypal traits.
\end{abstract}

Methods A total of 449 volunteers was included in this study, and autism-spectrum quotient (AQ), schizotypal personality questionnaire (SPQ), and attention network test (ANT) were used to measure autistic traits and schizotypal traits. The three independent attentional networks, including alerting network, orienting network, and executive control network, were also measured.

Results Autistic traits were associated with the orienting network, whereas schizotypal traits were associated with the orienting network and executive control network. Furthermore, attentional networks could be predicted by specific dimensions of autistic and schizotypal traits. AQ-attention switching [0.104 (-1.175- -0.025), $\mathrm{p}=0.041]$ and AQ-attention to detail [-0.097 (-0.798- -0.001$), \mathrm{p}=0.049]$ were significant predictors of orienting network and gender were significant predictor of executive network (Beta $=0.107 ; 95 \% \mathrm{CI}=-0.476-10.139$; $\mathrm{p}=0.031$ ). Whereas, schizotypal dimension "interpersonal" was a significant predictor of all three attentional networks [Alerting: 0.147 (-0.010-0.861), $\mathrm{p}=0.045$; Orienting: 0.147 (0.018-0.733), $\mathrm{p}=0.040$; Executive: 0.198 (0.215-1.309), $\mathrm{p}=0.006]$.

Conclusion This study demonstrated that autistic and schizotypal traits were associated with attentional networks. The specific dimensions of autistic and schizotypal traits could predict attentional networks. Nevertheless, the attentional networks predicted with these two traits were different.

Psychiatry Investig 2021;18(5):417-425

Key Words Autistic traits, Schizotypal traits, Attentional networks.

\section{INTRODUCTION}

In the current opinion, neurodevelopmental and neuropsychiatric disorders, e.g., autism spectrum disorder (ASD) and schizophrenia spectrum disorder (SSD), can reflect quantitative, dimensional traits distributed continuously in the general

Received: June 28, 2020 Revised: December 28, 2020 Accepted: February 16, 2021

$\triangle$ Correspondence: Kai Wang, MD, PhD

Department of Neurology, The First Affiliated Hospital, Anhui Medical University, No. 210, Jixi Road, Shushan District, Hefei City, Anhui Province, Hefei 230022, China

Tel: +86-055162923704, E-mail: wangkai1964@126.com

*These authors contributed equally to this work.

(c) This is an Open Access article distributed under the terms of the Creative Commons Attribution Non-Commercial License (https://creativecommons.org/licenses/bync/4.0) which permits unrestricted non-commercial use, distribution, and reproduction in any medium, provided the original work is properly cited. population. ${ }^{1,2}$ In other words, the traits of ASD/SSD are expressed with varying levels of severity in the neurotypical and subclinical populations. Evidence from behavioral ${ }^{3,4}$ and neuroimaging ${ }^{5,6}$ studies have increasingly shown that patients with ASD/SSD and individuals with autistic/schizotypal traits have common features and manifestations. Many researchers found that cognitive, emotional, and neurobiological impairments exist in individuals with autistic/schizotypal traits. Among these, seven to nine attentional function dysfunction was crucial.

Increasing evidence ${ }^{7-9}$ has confirmed that autistic or schizotypal traits are associated with changes in attentional function in typical individuals. Zhao et al..$^{10}$ found that individuals with higher autistic traits have a deficit in gaze-triggered attention. Other researchers subsequently confirmed that individuals with higher autistic traits shown a deficit that they 
cannot reasonably distribute attention to incoming non-facial $^{11}$ and facial stimuli. ${ }^{12}$ Several researchers revealed that individuals with higher autistic traits have deficits in global but enhance local attentional function. ${ }^{13}$ Studies also confirmed that higher autistic traits were associated with superior visual perceptual skills ${ }^{14}$ and search skills. ${ }^{15}$ In addition, Stotesbury et al. ${ }^{16}$ reported that individuals with higher schizotypal traits had greater performance decrements of attentional function when they needed to pay attention to lots of stimuli. Researchers also found that sustained attentional deficits were associated with individuals of higher schizotypal traits. ${ }^{17,18}$ According to previous studies, autistic traits were associated with the abnormal orienting attentional function. For example, individuals with higher autistic traits had stronger local attentional functions. They focus their attention on some more detailed places when observing objects. ${ }^{13}$ They seem unwilling to pay attention to places that contain social information. A study observed that individuals with high autistic traits had difficulty perceiving emotional information conveyed by the eyes. ${ }^{19}$ Schizotypal traits were also found to be associated with the abnormal orienting attentional function. The higher the schizotypal traits, the poorer the visual-spatial ability. ${ }^{20,21}$ However, some studies were unable to find that the schizotypal traits are related to abnormal directional attention..$^{22}$ Based on studies, it seems to be a consensus that schizotypal traits were associated with abnormal executive control attentional function, confirmed by numerous researches. ${ }^{23,24}$ The study also found that autistic traits were associated with abnormal executive control attentional function. ${ }^{25}$ Therefore, both autistic traits and schizotypal traits were associated with the atypical attentional function. However, it seemed that autistic traits were associated with abnormal orientation and executive control attentional function. In contrast, schizotypal traits were associated with abnormal executive control attentional function. There seems to be no consensus on whether schizotypal traits were associated with the abnormal orienting attentional function.

As mentioned above, we speculated that different attentional theories and paradigms might be an important reason for the mixed attentional function resulted in individuals with higher autistic traits. Posner and Petersen ${ }^{26}$ proposed an attentional theory and designed the attention network test (ANT). Fan et al. ${ }^{27}$ have applied it widely to assess attentional networks in typical and clinical populations. They separated the attentional function into three independent networks: alerting network, orienting network, and executive control network. Different brain structures dominated the three networks. The alerting network was characterized by producing and maintaining optimal vigilance to incoming stimuli. The orienting network focused on the ability to prioritize sensory input by selecting a modality or location. The executive network was concerned with the resolution of response conflicts between competing information. This study intended to use these promising techniques to assess whether the autistic and schizotypal traits are associated with attentional networks.

The autistic and schizotypal traits can reflect the features of ASD/SSD in typical individuals. Autistic traits include traits that reflect social communication, social interaction, and stereotyped behavior syndrome of ASD. Schizotypal traits include positive, negative, and disordered syndromes of SSD. The autism-spectrum quotient (AQ) and schizotypal personality questionnaire (SPQ) are the two most widely used psychometric self-report measures. These are used for screening individuals with a high level of autistic and schizotypal traits. ${ }^{28,29}$ The autistic traits measured by AQ that consisted of five dimensions: social skill, attention switching, attention to detail, communications, and imagination. ${ }^{30}$ The schizotypal traits measured by SPQ included three dimensions: cognitive-perceptual, interpersonal, and disorganized. ${ }^{31}$ Previous findings showed that different dimensions could cause different changes in cognitive function and imaging markers. For instance, Davis et al. ${ }^{32}$ found that individuals with higher scores in autistic traits "social communication" and "interaction" syndromes are associated with reduced looking at eyes. The individuals with higher scores in autistic traits "attention to detail" dimension relate to improved face recognition. Neuroimaging studies have also demonstrated that the striatocortical functional connectivity is associated with the "cognitive-perceptual" dimension rather than the "interpersonal" or "disorganized" dimension. ${ }^{33}$

According to current knowledge, no studies have been using ANT to examine the associations between autistic/schizotypal traits. Especially, different dimensions of these traits, and three independent attentional networks in the same typical individuals. Therefore, the primary goal of our study was to investigate the associations between attentional networks and autistic traits; also attentional networks and schizotypal traits. Furthermore, to find out the relations between the special dimension of autistic/schizotypal traits and the three independent attentional networks.

\section{METHODS}

\section{Standard protocol approval, consent}

The participants were informed in detail before the test specific implementation process. They signed informed consent after a verbal agreement to participate. The study was performed according to the declaration of Helsinki. The study protocol was approved by the Ethics Committee of Anhui Medical University, Hefei, Anhui, China (20180235). 


\section{Participants}

A total of 495 college students (243 males and 252 females) from Anhui Medical University was recruited in this study. After excluding 46 participants with missing data, 449 (224 males and 225 females) participated in this study (Table 1). The average ages of male and female participants were 18.79 1.09 years and $18.74 \pm 1.14$ years, respectively. All participants completed the AQ, the SPQ, and the ANT and received 50 RMB for their participation. All participants were right-handed and were able to understand the entire procedure of the experiment.

\section{Exclusion criteria}

The exclusion criteria were determined based on the standard criteria of this topic $c^{8,19}$ and those specifically related to this study. Based on the reports, the abnormal attentional function of current or previous psychological disorders and drug use were also considered while determining the exclu-

Table 1. Characteristics of subject and measurements in the study

\begin{tabular}{lc}
\hline \multicolumn{1}{c}{ Indexes } & Total $(\mathrm{N}=449)$ \\
\hline Characteristics & $224(49.89)$ \\
Gender (male), N (\%) & $20(19,20)$ \\
Age, years, median (quartile) & \\
Measurement & \\
AQ scores, median (quartile) & $116(110,122)$ \\
Total-AQ & $23(20,26)$ \\
AQ-S & $26(24,27)$ \\
AQ-AS & $24(21,26)$ \\
AQ-AD & $22(20,24)$ \\
AQ-C & $21(19,23)$ \\
AQ-IM & \\
SPQ scores, median (quartile) & $23(15,32)$ \\
Total-SPQ & $7(4,11)$ \\
SPQ-CP & $11(6.5,16)$ \\
SPQ-N & $4(2,7)$ \\
SPQ-D & \\
Attentional networks & $36(26,50)$ \\
Alerting, median (quartile) & $47.43 \pm 16.52$ \\
Orienting, mean $\pm S D$ & $109.7 \pm 24.83$ \\
Executive, mean $\pm S D$ & $527 \pm 48.99$ \\
Mean RT, mean $\pm S D$ & $0.97(0.97,0.98)$ \\
Accuracy, median (quartile) & \\
\hline
\end{tabular}

Total AQ: total Autism-Spectrum Quotient, Total SPQ: Schizotypal Personality Questionnaire, AQ-S: AQ-Social Skill, AQ-AS: AQAttention Switching, AQ-AD: AQ-Attention to Detail, AQ-C: AQCommunication, AQ-IM: AQ-Imagination, SPQ-CP: CognitivePerceptual Schizotypy, SPQ-N: Interpersonal Schizotypy, SPQ-D: Disorganization Schizotypy sion criteria. ${ }^{34,35}$ These exclusion criteria were 1) any serious physical illnesses, 2) current or previous psychological disorders such as anxiety and depression, 3 ) other axis I disorder(s), and 4) drug use.

\section{Measures}

\section{Autism-spectrum quotient}

The AQ was a questionnaire to test autistic traits in typical individuals, designed by Baron-Cohen et al. ${ }^{30}$ The AQ consisted of 50 items divided into five dimensions: social skill, attention switching, attention to detail, communication, and imagination. This measure was translated into Chinese by Zhang et al. ${ }^{36}$ for China. The Chinese version of AQ also had good reliability (0.89) and validity (0.81). As did by Zhang et al., ${ }^{36}$ we adopted the 4-point Likert scoring system in the present study. Scores ranged from 1 to 4 for items that showed autistic features: "definitely agree" scored 4 points, "slightly agree" scored 3 points, "slightly disagree" scored 2 points, and "definitely disagree" scored 1 point. The scale was inverted for the opposite items.

\section{Schizotypal personality questionnaire}

The SPQ consisted of 74 items, designed by Raine ${ }^{31}$ based on the DSM-III-R, to separate the person with schizotypal personality disorder from the general population. It usually consisted of three factors: positive symptoms, including hallucinations and delusions; negative symptoms, including alogia, apathy, and amotivation; and disorganized symptoms including thought disorder and bizarre behavior. Each item was rated on a dichotomous response of "yes" or "no" ("yes" scored 1 point, and "no" scored 0 points). The Chinese version of the SPQ translated by Chen et al. ${ }^{37}$ had good reliability (0.86) and validity (0.95).

\section{Attention network test}

The subjects observed the stimuli in front of a computer screen at a distance of approximately $70 \mathrm{~cm}$ and reacted using two buttons. The visual stimuli included a row of five black arrowheads placed horizontally at the center of the computer screen. The arrowheads pointed left or right. The background of the stimuli was gray. The subjects were required to focus on the middle arrowhead, whose direction was presented in three different ways: 1) same as the arrowheads on the sides (congruent condition), 2) opposite the arrowheads on the sides (incongruent condition), or 3) no arrowhead on the sides (neutral condition). The participants' task was to press the buttons as soon as possible, based on the direction of the middle arrowhead. The left index finger pressed the button when the middle arrowhead pointed to the left. The right in- 
dex finger pressed the corresponding button when the arrowhead pointed to the right. A fixation point "+" was first presented on the computer screen on each test, and then a cue “*” was given after $400 \mathrm{~ms}$ to 1,600 ms. There were four cues: 1) no cue: the second fixation point appeared directly at the center of the screen; 2) central cue: the location of cue "** was presented at the center of the screen; 3) double cue: the cue appeared above or below the fixation point but did not provide any spatial information; and 4) spatial cue: cues were displayed above or below the central fixation point, and the cue reminded the participant of the position of fixation. The entire ANT consisted of a 24-trial practice set and three experimental sets. Each experimental set consisted of 96 trials [48 trials: 4 (cue types) $\times 2$ arrow location (up and down) $\times 2$ arrow directions (left and right) $\times 3$ (ways of arrowheads), repeated twice]. The presentation of the trials was random. Participants were instructed to pay attention to a fixation point at the center of a computer screen and reacted when the screen changed. Figure 1 illustrates the task.

The relevant attentional network efficiencies were calculated as follows: The alerting network=the mean response time (RT) with no cue-the mean RT with double cues. The larger the value of the alerting network, the higher its efficiency. The orienting network=the mean RT with a center cue-the mean RT with a spatial cue. The larger the value of the orienting network, the higher its efficiency. The executive control network= the mean RT of the incongruent target condition-the mean $\mathrm{RT}$ of the congruent target condition. The larger the value of the executive control network, the lower its efficiency.

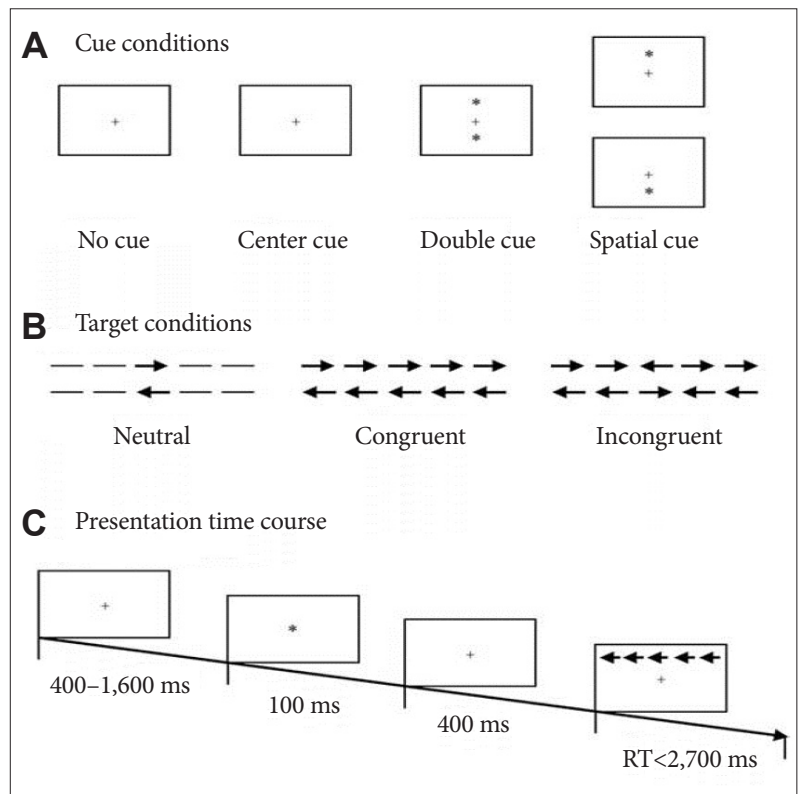

Figure 1. Experimental paradigm of the attention network test. A: The four cue conditions. B: The six stimuli used in the present experiment. C: An example of the procedure.

\section{Data analysis and statistics}

Data were expressed as mean $\pm \mathrm{SD}$, and statistical analysis was performed using SPSS V19.0 software (IBM Corp., Armonk, NY, USA). Pearson's correlation and Spearman's correlation tests were used to assess relationships between the AQ or SPQ scores and the three attentional networks' efficiencies. Descriptive statistics were used to measure data normality, and Spearman's correlation test was executed according to the normality of data in tables. Multiple linear regression analysis was performed to explore the dimension of autistic and schizotypal traits that could predict the three attentional networks. $\mathrm{p}<0.05$ was considered statistically significant.

\section{RESULTS}

\section{The correlation between autistic/schizotypal traits and ANT}

A positive relationship between the total AQ scores and the score of the orienting network was found $(r=0.115, \mathrm{p}<0.05)$ (Table 2). The total scores of SPQ are positively related to the scores of the alerting network and orienting network $(r=0.061$, $\mathrm{p}<0.05 ; \mathrm{r}=0.099, \mathrm{p}<0.05$ ) (Table 3).

As for the relationship between the various dimensions of autistic traits and attentional networks, a positive relation between the score of the orienting network and "social skill, communication and imagination" dimensions were found ( $\mathrm{r}=$ $0.183, \mathrm{p}<0.01 ; \mathrm{r}=0.175, \mathrm{p}<0.01 ; \mathrm{r}=0.125, \mathrm{p}<0.05)$. A negative relation between the score of the orienting network and "attention to detail" dimension was found $(\mathrm{r}=-0.110, \mathrm{p}<0.05)$ (Table 2).

A positive relationship between the score of the orienting network, executive control network, and the scores of 'interpersonal' dimensions of schizotypal traits was found $(\mathrm{r}=0.182$, $\mathrm{p}<0.01 ; \mathrm{r}=0.123, \mathrm{p}<0.05)$ (Table 3).

\section{The multiple regression analysis of ANT}

We performed a multiple regression analysis of all dimensions of autistic, schizotypal traits and the three networks. We found that "interpersonal" dimension was a significant predictor of all three networks [alerting: $0.147(-0.010-0.861), \mathrm{p}=$ 0.045 ; orienting: 0.147 (0.018-0.733), $\mathrm{p}=0.040$; executive: 0.198 (0.215-1.309), $\mathrm{p}=0.006]$. Besides, $\mathrm{AQ}$-attention switching [0.104 (-1.175- -0.025), $\mathrm{p}=0.041]$ and $\mathrm{AQ}$-attention to detail $\left[-0.097\left(-0.798^{-}-0.001\right), \mathrm{p}=0.049\right]$ were significant predictors of orienting network, and gender was a significant predictor of Executive network (Beta $=0.107 ; 95 \% \mathrm{CI}=-0.476-10.139$; $\mathrm{p}=0.031)$ (Table 4). 
Table 2. Correlation between autistic traits and ANT

\begin{tabular}{|c|c|c|c|c|c|c|c|c|c|c|c|}
\hline & Total AQ & AQ-S & AQ-AS & AQ-AD & AQ-C & AQ-IM & Alerting & Orienting & Executive & Mean RT & Accuracy \\
\hline Total-AQ & 1 & & & & & & & & & & \\
\hline AQ-S & $0.712^{* *}$ & 1 & & & & & & & & & \\
\hline AQ-AS & $0.494^{* *}$ & $0.327^{* *}$ & 1 & & & & & & & & \\
\hline AQ-AD & $0.275^{* *}$ & $-0.131^{* *}$ & -0.072 & 1 & & & & & & & \\
\hline AQ-C & $0.677^{* *}$ & $0.507^{* *}$ & $0.255^{* *}$ & -0.057 & 1 & & & & & & \\
\hline AQ-IM & $0.540^{* *}$ & $0.263^{* *}$ & $0.099^{*}$ & -0.057 & $0.270^{* *}$ & 1 & & & & & \\
\hline Alerting & 0.020 & 0.066 & 0.072 & -0.028 & 0.006 & -0.021 & 1 & & & & \\
\hline Orienting & $0.115^{*}$ & $0.183^{* *}$ & 0.009 & $-0.110^{*}$ & $0.175^{* *}$ & $0.125^{*}$ & 0.068 & 1 & & & \\
\hline Executive & 0.026 & 0.005 & 0.072 & 0.039 & 0.035 & 0.006 & -0.054 & -0.001 & 1 & & \\
\hline Mean RT & 0.063 & $0.107^{*}$ & 0.021 & -0.078 & 0.073 & $0.104^{*}$ & 0.015 & $0.244^{* *}$ & $0.237^{* *}$ & 1 & \\
\hline Accuracy & -0.033 & -0.037 & -0.013 & -0.028 & -0.041 & 0.047 & $-0.099 *$ & 0.033 & $-0.110^{* *}$ & $0.422^{* *}$ & 1 \\
\hline
\end{tabular}

${ }^{*} \mathrm{p}<0.05,{ }^{* *} \mathrm{p}<0.01$. ANT: attention network test, Total AQ: total Autism-Spectrum Quotient, AQ-S: AQ-Social Skill, AQ-AS: AQ-Attention Switching, AQ-AD: AQ-Attention to Detail, AQ-C: AQ-Communication, AQ-IM: AQ-Imagination

Table 3. Correlation between schizotypal traits and ANT

\begin{tabular}{|c|c|c|c|c|c|c|c|c|c|}
\hline & Total-SPQ & SPQ-P & SPQ-IN & SPQ-D & Alerting & Orienting & Executive & Mean RT & Accuracy \\
\hline SPQ-total & 1 & & & & & & & & \\
\hline SPQ-P & $0.828^{* *}$ & 1 & & & & & & & \\
\hline SPQ-IN & $0.853^{* *}$ & $0.501^{* *}$ & 1 & & & & & & \\
\hline SPQ-D & $0.790^{* *}$ & $0.621^{* *}$ & $0.523^{* *}$ & 1 & & & & & \\
\hline Alerting & 0.061 & 0.032 & 0.089 & 0.008 & 1 & & & & \\
\hline Orienting & $0.099^{*}$ & -0.007 & $0.182^{* *}$ & 0.054 & 0.068 & 1 & & & \\
\hline Executive & $0.111^{*}$ & 0.081 & $0.123^{* *}$ & 0.086 & -0.054 & -0.001 & 1 & & \\
\hline Mean RT & 0.005 & -0.037 & 0.069 & -0.013 & 0.015 & $0.244^{* *}$ & $0.237^{* *}$ & 1 & \\
\hline Accuracy & -0.053 & -0.026 & -0.053 & -0.055 & $-0.099^{*}$ & 0.033 & $-0.110^{* *}$ & $0.422^{* *}$ & 1 \\
\hline
\end{tabular}

${ }^{*} \mathrm{p}<0.05,{ }^{* *} \mathrm{p}<0.01$. ANT: attention network test, Total SPQ: Schizotypal Personality Questionnaire, SPQ-CP: Cognitive-Perceptual Schizotypy, SPQ-IN: Interpersonal Schizotypy, SPQ-D: Disorganization Schizotypy

\section{DISCUSSION}

The present study used AQ, SPQ, and ANT to measure autistic traits, schizotypal traits, and attentional network relationships. The study suggested that autistic traits were associated with the orienting network, and schizotypal traits are correlated with all the three attentional networks. Moreover, our study demonstrated that the "communication" dimension of autistic traits could predict the efficiency of the orienting network. The "interpersonal" dimension of schizotypal traits could predict the efficiency of the three attentional networks.

In previous studies, researchers found that the attentional function changes were core characteristics of patients with ASD and individuals with higher autistic traits. ${ }^{13,38}$ Keehn et al..$^{39}$ found that children and teenagers with ASD had a lower efficiency in orienting networks, which was not entirely consistent with our research. This may be because participants were ASD patients instead of individuals with higher autistic traits. Our study showed that total autistic trait scores were negatively associated with orienting network efficiency. Individuals with higher scores in total autistic traits had higher efficiency in the orienting network. According to studies, individuals with high autistic traits also had impaired attentional function. These including delayed attention to others' eyes ${ }^{19}$ and difficulty recognizing emotions ${ }^{40}$ indicating they had a deficit in attention to the social stimulus. This study revealed that individuals with high autistic traits had a deficit orienting functional efficiency, which was inconsistent with previous studies. It was due to arrows belonging to non-social information stimuli. Previous studies found that autistic traits were mostly associated with impaired social stimuli. However, other studies found that individuals with high autistic traits were more efficient at non-social stimuli and responded more quickly to non-social information stimuli. ${ }^{14,15}$ Several earlier studies using the ANT paradigm reported reduced effectiveness in the orienting function of arrow information among 


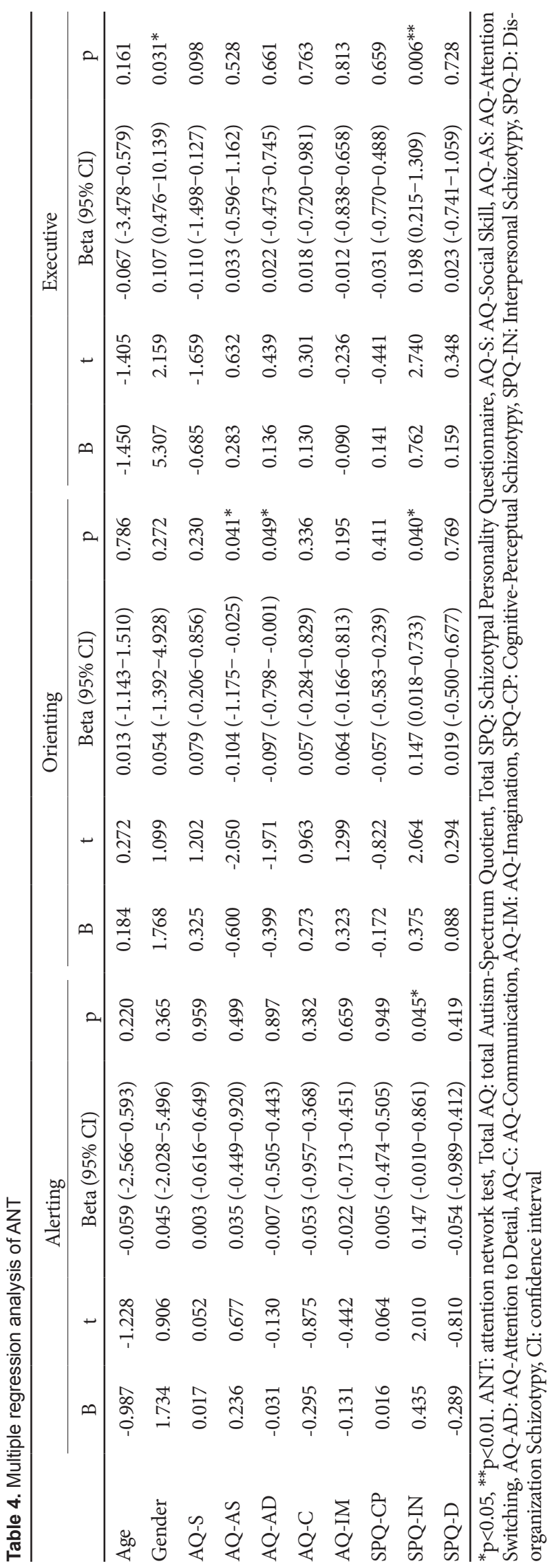

ASD patients. ${ }^{39,41,42}$ The subjects of the previous research were ASD children with significantly impaired social function. In contrast, our study enrolled young people admitted to a university and without impairing social function. These collegeeducated individuals had higher IQs, better social skills, and better spatial recognition than those with ASD.

In exploring the relationship between the special autistic dimension and orienting network, our study found that the autistic dimensions "social skill, communication, and imagination" were positively associated with the efficiency of the orienting network. However, our study suggested that individuals with higher scores of autistic dimensions, "attention to detail" are negatively associated with the orienting network's efficiency. Our study results showed that only the individuals with higher scores in autistic dimension "attention to detail" exhibited the deficit in the orienting network similar to that of ASD patients. New diagnostic and statistical manual of mental disorders, 5th edition (DSM-5) emphasizes the different dimensional characteristics of ASD patients. ${ }^{43}$ An increasing evidence proved that different dimensions of autistic traits were associated with different cognition features. ${ }^{44,45}$ In our study, different dimensional traits of participants' may have caused the mixed results of attentional function changes.

Several studies found attentional disability in individuals with higher schizotypal traits, which was consistent with the present study. ${ }^{16-18}$ However, no studies investigated which attentional network had deficits in individuals with higher schizotypal traits. Studies focused on schizotypal traits and attentional functions noticed that higher schizotypal traits were associated with the impaired attentional function. ${ }^{16,17}$ For example, when normal people deal with external stimuli, their ability to deal with the stimuli decreases as the stimuli increase. In contrast, individuals with higher schizotypal traits experienced an even greater decline in their ability to deal with more stimuli. ${ }^{16}$ Some studies found that individuals with high schizotypal traits had impaired sustained attentional function, which made them unable to pay attention for a long time. ${ }^{17}$ Similarly, this study revealed that schizotypal traits were associated with poor executive control efficiency. Previous studies have also shown impaired executive control attentional function in patients with schizophrenia. ${ }^{46}$ This study confirmed impaired attentional function in individuals with high schizotypal traits among normal populations. Schizotypal traits affected normal populations' attentional function. The higher the trait score, the worse the executive control attentional function.

To our surprise, there was an enhanced efficiency in alerting and orienting networks of individuals with higher schizotypal traits. Previous findings suggested that individuals with higher schizotypal traits had deficits in the auditory alertness ${ }^{47}$ and attentional alertness. ${ }^{17,18}$ Also, patients with SSD had an 
impairment in the orienting network. ${ }^{48,49}$ The results of our study were inconsistent with the previous results as mentioned above. Individuals with higher schizotypal traits may have shown deficits in some cognitive tasks in most previous studies. However, it does not mean that these people were only associated with impaired cognitive function. ${ }^{50}$ The most significant difference between our study and prior researches is the task and volunteers. Participants of this study were more likely to have sufficient cognitive strength to achieve a college education than the general SSD patient group.

Schizotypal traits can be grouped into separate cognitiveperceptual, interpersonal, and disorganized dimensions. Our study showed that the schizotypal dimension "interpersonal" could predict alterations in the attentional networks. The behavioral and imaging performance of individuals with schizotypal traits was associated with dimensions. Also, individuals with higher cognitive-perceptual schizotypal dimensions had a talent for creativity. ${ }^{51-53}$ However, individuals with higher interpersonal schizotypal dimensions had deficits in episodic memory. ${ }^{54}$ The neural activities of individuals with higher schizotypal scores in cognitive-perceptual or interpersonal dimensions were different. ${ }^{55}$ The different cognition function performances may have been caused by different underlying neural activities of individuals with higher schizotypal traits in the "cognitive-perceptual," "interpersonal" dimension.

Autistic traits and schizotypal traits interact. Many ASD patients had been diagnosed with schizophrenia, ${ }^{2,56}$ and had impaired attentional function, indicating that they may share a common pathogenesis. This study revealed that autistic traits and schizotypal traits had hyperfunction of orienting attention. It had two sides, and it made us more capable in several aspects, such as quickly finding some external stimuli. ${ }^{14,15}$ However, it also had inevitably led us to be more sensitive, less able to pay attention, and more vulnerable to external stimuli. Both patients with ASD and schizophrenia were sensitive to external information. They were prone to anger or fear, ${ }^{57-59}$ leading to withdrawal from social interactions and thus exacerbates social dysfunction. Further investigation of the similarity between autistic traits and schizotypal deficits may help better understand the clinical symptoms of ASD patients, similar to those of schizophrenia. Therefore, providing some potential benefits of behavioral intervention.

The present study had several limitations. Firstly, recruited volunteers were confined to college students only. Autistic traits and schizotypal traits may have affected their college admission. Previous studies have shown that autistic traits and schizotypal traits can affect an individual's income and social status. Although no reviews have discussed the relationship between autistic traits and schizotypal traits and college admission, it is likely to impact. Secondly, participants with other axis I disorder(s) was not recruited, considering the standard criteria for this topic ${ }^{8,19}$ and target participants in this initial study. The study did not measure other characteristics that may have affected the attentional function, such as anxiety, depression, obsessive-compulsive disorder (OCD), attention deficit, and hyperactivity disorder (ADHD). However, the subjects were asked if they had any of these diseases. Thirdly, the scales used in this study with good reliability and validity were AQ and SPQ based on this topic's standard criteria. ${ }^{60,61}$ Therefore, other scales such as BPRS, SCL-90, PANSS, and YBOCS were not considered in this initial study. Besides, the arrow stimulus used in this study was non-social stimuli. The attentional function of patients with autism towards non-social stimuli and social stimuli were different. This may have resulted in an inability to fully explore the relationship between autistic traits and schizotypal traits, and the attentional function.

\section{Conclusion}

This study demonstrated that autistic and schizotypal traits were associated with attentional networks. Specific dimensions of autistic and schizotypal traits can predict different attentional networks.

\section{Acknowledgments}

Many thanks to all participants. The authors also want to acknowledge the valuable help from Yaoting Sun.

This work was supported by the natural science foundation of China $(31800909,91432301)$.

\section{Conflicts of Interest}

The authors have no potential conflicts of interest to disclose.

\section{Author Contributions}

Conceptualization: Kai Wang, Long Zhang. Data curation: Wanling Huang. Formal analysis: Wanling Huang. Funding acquisition: Kai Wang, Long Zhang. Investigation: Wanling Huang. Methodology: Wanling Huang. Project: Wanling Huang. Administration: Kai Wang. Resources: Fangfang Chen. Software: Yaoting Sun. Supervision: Kai Wang. Validation: Wanling Huang. Visualization: Wanling Huang. Writing_original draft: Wanling Huang. Writing_-review \& editing: Wanling Huang, Kai Wang, Long Zhang.

\section{ORCID iDs}

$\begin{array}{ll}\text { Wanling Huang } & \text { https://orcid.org/0000-0002-0551-4479 } \\ \text { Long Zhang } & \text { https://orcid.org/0000-0003-1988-7951 } \\ \text { Yaoting Sun } & \text { https://orcid.org/0000-0002-2277-2058 } \\ \text { Fangfang Chen } & \text { https://orcid.org/0000-0003-0582-7245 } \\ \text { Kai Wang } & \text { https://orcid.org/0000-0002-6197-914X }\end{array}$

\section{REFERENCES}

1. Constantino JN, Todd RD. Intergenerational transmission of subthreshold autistic traits in the general population. Biol Psychiatry 2005;57: 655-660.

2. St Pourcain B, Robinson EB, Anttila V, Sullivan BB, Maller J, Golding J, et al. ASD and schizophrenia show distinct developmental profiles in common genetic overlap with population-based social communication difficulties. Mol Psychiatry 2018;23:263-270. 
3. Madipakkam AR, Rothkirch M, Dziobek I, Sterzer P. Access to awareness of direct gaze is related to autistic traits. Psychol Med 2018;27:1-7.

4. Ferri F, Ambrosini E, Costantini M. Spatiotemporal processing of somatosensory stimuli in schizotypy. Sci Rep 2016;6:38735.

5. Rashid B, Blanken LME, Muetzel RL, Miller R, Damaraju E, Arbabshirani $\mathrm{MR}$, et al. Connectivity dynamics in typical development and its relationship to autistic traits and autism spectrum disorder. Hum Brain Mapp 2018;39:3127-3142.

6. DeRosse P, Nitzburg GC, Ikuta T, Peters BD, Malhotra AK, Szeszko PR. Evidence from structural and diffusion tensor imaging for frontotemporal deficits in psychometric schizotypy. Schizophr Bull 2015;41:104114.

7. Blain SD, Peterman JS, Park S. Subtle cues missed: Impaired perception of emotion from gait in relation to schizotypy and autism spectrum traits. Schizophr Res 2017;183:157-160.

8. Karvelis P, Seitz AR, Lawrie SM, Serie's P. Autistic traits, but not schizotypy, predict increased weighting of sensory information in Bayesian visual integration. eLife 2018;7:e34115.

9. Ford TC, Woods W, Crewther DP. Mismatch field latency, but not power, may mark a shared autistic and schizotypal trait phenotype. Int J Psychophysiol 2017;116:60-67.

10. Zhao S, Uono S, Yoshimura S, Toichi M. Is impaired joint attention present in non-clinical individuals with high autistic traits? Mol Autism 2015; 6:67.

11. English MC, Maybery MT, Visser TA. Individuals with autistic-like traits show reduced lateralization on a greyscales task. J Autism Dev Disord 2015;45:3390-3395.

12. Dundas EM, Best CA, Minshew NJ, Strauss MS. A lack of left visual field bias when individuals with autism process faces. J Autism Dev Disord 2012;42:1104-1111.

13. Laycock R, Chan D, Crewther SG. Attention orienting in response to non-conscious hierarchical arrows: individuals with higher autistic traits differ in their global/local bias. Front Psychol 2017;8:23.

14. Sabatino DiCriscio A, Troiani V. Brief Report: autism-like traits are associated with enhanced ability to disembed visual forms. J Autism Dev Disord 2017;47:1568-1576.

15. Van der Hallen R, Chamberlain R, de-Wit L, Wagemans J. Superior disembedding in children with ASD: new tests using abstract, meaningful, and 3D contexts. J Autism Dev Disord 2018;48:2478-2489.

16. Stotesbury H, Gaigg SB, Kirhan S, Haenschel C. The influence of schizotypal traits on attention under high perceptual load. Schizophr Res Cogn 2017;11:6-10.

17. Chan RC, Wang Y, Cheung EF, Cui J, Deng Y, Yuan Y, et al. Sustained attention deficit along the psychosis proneness continuum: a study on the Sustained Attention to Response Task (SART). Cogn Behav Neurol 2009; 22:180-185.

18. Alvarez-Moya EM, Barrantes-Vidal N, Navarro JB, Subira S, Obiols JE. Exophenotypical profile of adolescents with sustained attention deficit: a 10-year follow-up study. Psychiatry Res 2007;153:119-130.

19. Kleberg JL, Högström J, Nord M, Bölte S, Serlachius E, Falck-Ytter T. Autistic traits and symptoms of social anxiety are differentially related to attention to others' eyes in social anxiety disorder. J Autism Dev Disord 2017;47:3814-3821.

20. Gooding DC, Tallent KA. Spatial, object, and affective working memory in social anhedonia: an exploratory study. Schizophr Res 2003;63: 247-260.

21. Robles O, Blaxton T, Adami H, Arango C, Thaker G, Gold J. Nonverbal delayed recognition in the relatives of schizophrenia patients with or without schizophrenia spectrum. Biol Psychiatry 2008;63:498-504.

22. Gouzoulis-Mayfrank E, Balke M, Hajsamou S, Ruhrmann S, SchultzeLutter F, Daumann J, et al. Orienting of attention in unmedicated patients with schizophrenia, prodromal subjects and healthy relatives. Schizophr Res 2007;97:35-42.

23. López SG, Fuster JI, Reyes MM, Collazo TM, Quiñones RM, Berazain $\mathrm{AR}$, et al. Attentional network task in schizophrenic patients and theirs unaffected first degree relatives: a potential endofenotype. Actas Esp Psiquiatr 2011;39:32-44.

24. Chang TG, Lee IH, Chang CC, Yang YK, Huang SS, Chen KC, et al. Poorer Wisconsin card-sorting test performance in healthy adults with higher positive and negative schizotypal traits. Psychiatry Clin Neurosci 2011;65:596-599.

25. Mundy P, Sullivan L, Mastergeorge AM. A parallel and distributed-processing model of joint attention, social cognition and autism. Autism Res 2009;2:2-21.

26. Posner MI, Petersen SE. The attention system of the human brain. Annu Rev Neurosci 1990;13:25-42.

27. Fan J, McCandliss BD, Sommer T, Raz A, Posner MI. Testing the efficiency and independence of attentional networks. J Cogn Neurosci 2002;14:340-347.

28. Kuno M, Hirano Y, Nakagawa A, Asano K, Oshima F, Nagaoka S, et al. White matter features associated with autistic traits in obsessive-compulsive disorder. Front Psychiatry 2018;9:216.

29. Rössler J, Unterassner L, Wyss T, Haker H, Brugger P, Rössler W, et al. Schizotypal traits are linked to dopamine-induced striato-cortical decoupling: a randomized double-blind placebo-controlled study. Schizophr Bull 2019;45:680-688.

30. Baron-Cohen S, Wheelwright S, Skinner R, Martin J, Clubley E. The autism-spectrum quotient (AQ): evidence from Asperger syndrome/ high-functioning autism, males and females, scientists and mathematicians. J Autism Dev Disord 2001;31:5-17.

31. Raine A. The SPQ: a scale for the assessment of schizotypal personality based on DSM-III-R criteria. Schizophr Bull 1991;17:555-564.

32. Davis J, McKone E, Zirnsak M, Moore T, O'Kearney R, Apthorp D, et al. Social and attention-to-detail subclusters of autistic traits differentially predict looking at eyes and face identity recognition ability. Br J Psychol 2017;108:191-219.

33. Wang Y, Ettinger U, Meindl T, Chan RCK. Association of schizotypy with striatocortical functional connectivity and its asymmetry in healthy adults. Hum Brain Mapp 2018;39:288-299.

34. Eysenck MW, Derakshan N, Santos R, Calvo MG. Anxiety and cognitive performance: attentional control theory. Emotion 2007;7:336-353.

35. Loeffler LAK, Satterthwaite TD, Habel U, Schneider F, Radke S, Derntl B. Attention control and its emotion-specific association with cognitive emotion regulation in depression. Brain Imaging Behav 2019;13:17661779.

36. Zhang L, Sun Y, Chen F, Wu D, Tang J, Han X, et al. Psychometric properties of the Autism-Spectrum Quotient in both clinical and non-clinical samples: Chinese version for mainland China. BMC Psychiatry 2016; 16:213.

37. Chen WJ, Hsiao CK, Lin CC. Schizotypy in community samples: the three-factor structure and correlation with sustained attention. J Abnorm Psychol 1997;106:649-654.

38. Kanat M, Spenthof I, Riedel A, van Elst LT, Heinrichs M, Domes G. Restoring effects of oxytocin on the attentional preference for faces in autism. Transl Psychiatry 2017;7:e1097.

39. Keehn B, Lincoln AJ, Müller RA, Townsend J. Attentional networks in children and adolescents with autism spectrum disorder. J Child Psychol Psychiatry 2010;51:1251-1259.

40. Bothe E, Palermo R, Rhodes G, Burton N, Jeffery L. Expression recognition difficulty is associated with social but not attention-to-detail autistic traits and reflects both alexithymia and perceptual difficulty. J Autism Dev Disord 2019;49:4559-4571.

41. Hames EC, Rajmohan R, Fang D, Anderson R, Baker M, Richman DM, et al. Attentional networks in adolescents with high-functioning autism: an fMRI investigation. Open Neuroimag J 2016;10:102-110.

42. Mutreja R, Craig C, O’Boyle MW. Attentional network deficits in children with autism spectrum disorder. Dev Neurorehabil 2016;19:389397.

43. American Psychiatric Association. Diagnostic and Statistical Manual of Mental Health Disorders (DSM-5). 5th Ed. Washington, DC: American 
Psychiatric Publishing; 2013

44. Palmer CJ, Paton B, Enticott PG, Hohwy J. 'Subtypes' in the presentation of autistic traits in the general adult population. J Autism Dev Disord 2015;45:1291-1301.

45. Davis J, McKone E, Zirnsak M, Moore T, O’Kearney R, Apthorp D, et al. Social and attention-to-detail subclusters of autistic traits differentially predict looking at eyes and face identity recognition ability. Br J Psychol 2017;108:191-219.

46. Orellana G, Slachevsky A, Peña M. Executive attention impairment in first-episode schizophrenia. BMC Psychiatry 2012;12:154.

47. Seidman LJ, Pousada-Casal A, Scala S, Meyer EC, Stone WS, Thermenos HW, et al. Auditory vigilance and working memory in youth at familial risk for schizophrenia or affective psychosis in the Harvard adolescent family high risk study. J Int Neuropsychol Soc 2016;22:10261037.

48. Backes V, Kellermann T, Voss B, Krämer J, Depner C, Schneider F, et al. Neural correlates of the attention network test in schizophrenia. Eur Arch Psychiatry Clin Neurosci 2011;261:S155-S160.

49. Nestor PG, Kubicki M, Spencer KM, Niznikiewicz M, McCarley RW, Shenton ME. Attentional networks and cingulum bundle in chronic schizophrenia. Schizophr Res 2007;90:308-315.

50. Claridge G, Clark K, Davis C. Nightmares, dreams, and schizotypy. Br J Clin Psychol 1997;36:377-386.

51. Fejes N, Rózsa S, Must A. Schizotypal traits and verbal creativity. Ideggyogy Sz 2018;71:113-125.

52. Lindell AK. On the interrelation between reduced lateralization, schizotypy, and creativity. Front Psychol 2014;5:813.

53. Park HR, Kirk IJ, Waldie KE. Neural correlates of creative thinking and schizotypy. Neuropsychologia 2015;73:94-107.

54. Sahakyan L, Kwapil TR. Episodic memory retrieval is impaired in negative schizotypy under fast response deadline. Schizophr Res 2018;201: 167-171.

55. Yan C, Wang Y, Su L, Xu T, Yin DZ, Fan MX, et al. Differential mesolimbic and prefrontal alterations during reward anticipation and consummation in positive and negative schizotypy. Psychiatry Res Neuroimaging 2016;254:127-136.

56. Zheng Z, Zheng P, Zou X. Association between schizophrenia and autism spectrum disorder: a systematic review and meta-analysis. Autism Res 2018;11:1110-1119.

57. Baskak NS, Özateș ME, Herdi O, Sonel E, Ulusan A, Baskak B. The relation between functional anatomy of the face and threat perception evoked by facial expression of anger in schizophrenia. Noro Psikiyatr Ars 2019;56:7-12.

58. Leung RC, Pang EW, Brian JA, Taylor MJ. Happy and angry faces elicit atypical neural activation in children with autism spectrum disorder. Biol Psychiatry Cogn Neurosci Neuroimaging 2019;4:1021-1030.

59. Malaia E, Cockerham D, Rublein K. Visual integration of fear and anger emotional cues by children on the autism spectrum and neurotypical peers: An EEG study. Neuropsychologia 2019;126:138-146.

60. Amoruso L, Finisguerra A, Urgesi C. Autistic traits predict poor integration between top-down contextual expectations and movement kinematics during action observation. Sci Rep 2018;8:16208.

61. Moreno-Samaniego L, Gaviria AM, Vilella E, Valero J, Labad A. Schizotypal traits and cognitive performance in siblings of patients with psychosis. Psychiatry Res 2017;258:551-556. 